\title{
PARTICLE-IN-CELL SIMULATIONS OF THE HIGH CURRENT EXPERIMENT*
}

\author{
C.M. Celata, F.M. Bieniosek, L. Prost, and P.A. Seidl, Lawrence Berkeley National Laboratory, \\ Berkeley, CA, USA, and the Heavy Ion Fusion Virtual National Laboratory \\ A. Friedman and D.P. Grote, Lawrence Livermore National Laboratory, Livermore, CA, USA, and the \\ Heavy Ion Fusion Virtual National Laboratory
}

\begin{abstract}
The particle-in-cell code WARP has been used to simulate beam dynamics for the intense ion beam of the High Current Experiment. First a study was done of the dynamic aperture of the alternating-gradient electrostatic quadrupole lattice of the experiment, including nonlinearity due to image forces and imperfections of the focusing lattice field. It was found that particle loss, rather than emittance growth, determined the usable aperture. Simulations of transport in the High Current Experiment were then performed, and the results compared to measured data. We present the results of both of these studies.
\end{abstract}

\section{INTRODUCTION}

The High Current Experiment (HCX) [1], a Heavy Ion Fusion Virtual National Laboratory experiment located at LBNL, employs a driver-scale beam to investigate transport limits for heavy ion inertial fusion induction linac drivers. The beam is a coasting $\mathrm{K}^{+}$beam, which is transported through an alternating gradient FODO lattice. At present the current, I, is $185 \mathrm{~mA}$ at $1 \mathrm{MeV}$, and the lattice consists of a ten-electrostatic-quadrupole alternating-gradient system, followed by four magnetic quadrupoles. Expected eventual parameters are $555 \mathrm{~mA}$ at $1.8 \mathrm{MeV}$.

In this report, the dynamic aperture of the HCX electrostatic lattice, which is expected to be similar to an electrostatic-focus section of a driver, is investigated, using the transverse 2D version of the particle-in-cell (PIC) simulation. code WARP [2]. The dynamic aperture is an important cost factor for a heavy ion fusion power plant accelerator. In the driver, multiple $(\sim 100)$ beams will be accelerated in parallel through common induction cores. An increase in the aperture necessary for each beam will have a large impact on the radius of the induction core and focusing structures, and thus on the size, weight, and cost of the accelerator.

Two sets of results are reported here. In the first, scaling studies were performed with an initially semigaussian beam, varying the current and undepressed betatron tune of the lattice, in order to find the dynamic aperture of the system for an idealized beam. In the second, the simulation was initialized with a particle distribution function synthesized using data from the upstream end of the HCX

\footnotetext{
* Supported by the Office of Fusion Energy Science, US DOE, contract numbers DE-AC03-76SF00098 and W-7405-Eng-48.
}

experiment, just after the matching section, in order to compare the results with experiment.

\section{SIMULATION MODEL FOR DYNAMIC APERTURE SCALING STUDIES}

We discuss first the calculations made to determine the dynamic aperture of an alternating gradient focusing lattice assumed to consist of the HCX electrostatic lattice, lengthened to 50 lattice periods in order to have sensitivity to longer-length-scale effects. The quadrupole structure is shown in Fig. 1. The quadrupole electrode radius was selected to eliminate the dodecapole field component (i.e., electrode radius $=8 / 7 \mathrm{x}$ aperture radius). The HCX focusing fields were modelled at each $\mathrm{z}$ by means of multipole moments derived from a 3D solution of the Poisson equation for the HCX quadrupoles, which included in the calculation the cylindrical quadrupole electrodes and the charged plates supporting them. Moments giving potential values greater than $1.5 \%$ of the quadrupole potential at a radius of $85 \%$ of the quadrupole aperture (aperture $=2.3 \mathrm{~cm}$, given by the quadrupole electrode surface) were included, with a $\mathrm{z}$ resolution of $0.85 \mathrm{~mm}$. Image forces for the same focusing structure, assuming perfect conductors, were calculated at each timestep using a capacitive matrix technique. A square conducting box at $4.9 \mathrm{~cm}$ bounded the $512 \times 512$ cell computational grid. 80 timesteps per lattice period (lattice period $=0.4352 \mathrm{~m}$ ) were used, giving adequate resolution of the fringe fields.

The simulations followed a transverse slice of the beam through 50 periods of alternating gradient lattice. The beam energy was assumed to be $1.8 \mathrm{MeV}$. The initial emittance was set to 5 times the thermal emittance of a $0.1 \mathrm{eV}, 5 \mathrm{~cm}$ radius source for a beam with $\mathrm{I}=576 \mathrm{~mA}$. It was then scaled with the square root of the current for other values of the current, simulating the effect of changing the source diameter for the same diode/injector. This scaling neglects differences in injector aberrations with beam size. Since ultimately the injector would be designed for the desired current, this seems to be a reasonable approximation. The emittance values used correspond to a space-chargedominated beam, with phase advance per lattice period, $\square$, a factor of 7-9 below its undepressed value.

An initially semigaussian distribution function was used for the scaling study because the driver is expected to have a 